\title{
EXPLICIT BOUNDS ON GAMIDOV TYPE INTEGRAL INEQUALITIES
}

\author{
B. G. PACHPATTE
}

\begin{abstract}
The aim of this paper is to establish explicit upper bounds on certain Gamidov type integral inequalities which can be used as convenient tools in some applications. The discrete analogues and applications are given to illustrate the usefulness of one of our results.
\end{abstract}

\section{Introduction}

In [4] Sh. G. Gamidov, while studying the boundary value problem for higher order differential equations, initiated the study of obtaining explicit upper bounds on the integral inequalities of the forms

$$
u(t) \leq c+\int_{\alpha}^{t} a(s) u(s) d s+\int_{\alpha}^{\beta} b(s) u(s) d s,
$$

for $t \in[\alpha, \beta]$, under some suitable conditions on the functions involved in (1.1). The use of such inequalities (usually called Gronwall or Gronwall-Bellman type inequalities) in the study of differential equations dates back at least to G.Peano in 1885. For detailed account on inequalities of the type (1.1) and some recent results, see [2, 3, 6-10]. In the present paper we shall consider the problem of obtaining explicit upper bounds on the general versions of (1.1) which can be used as tools in the study of qualitative behavior of solutions of certain classes of integral equations. The discrete analogues of the main results and applications of one of our results are also given.

\section{Statement of Results}

In what follows, $R$ denotes the set of real numbers and $R_{+}=[0, \infty), I=[\alpha, \beta]$ are the given subsets of $R$. Let $Z$ be the set of integers and for $\alpha, \beta \in Z, \alpha \leq \beta$, let $N_{\alpha, \beta}=\{n \in Z: \alpha \leq n \leq \beta\}$. We denote by $E=\left\{(t, s) \in I^{2}: \alpha \leq s \leq t \leq \beta\right\}$ and $H=\left\{(n, s) \in N_{\alpha, \beta}^{2}: \alpha \leq s \leq n \leq \beta\right\}$. For any function $w$ defined on $Z$, define the operator $\Delta$ by $\Delta w(n)=w(n+1)-w(n)$. We use the usual conventions that the empty sums and products are taken to be 0 and 1 respectively

Received and revised May 13, 2004.

2000 Mathematics Subject Classification. 26D10, 26D20.

Key words and phrases. Explicit bounds, Gamidov type integral inequalities, discrete analogues, partial derivative, Volterra-Fredholm integral equation. 
Our main results are established in the following theorem.

Theorem 1. Let $u, a, b, c, f, g \in C\left(I, R_{+}\right)$.

$\left(\mathrm{a}_{1}\right)$ Let $a(t)$ be continuously differentiable on $I, a(t) \geq 0$ and

$$
u(t) \leq a(t)+\int_{\alpha}^{t} b(s) u(s) d s+\int_{\alpha}^{\beta} c(s) u(s) d s
$$

for $t \in I$. If

$$
p_{1}=\int_{\alpha}^{\beta} c(s) \exp \left(\int_{\alpha}^{s} b(\sigma) d \sigma\right) d s<1
$$

then

$$
u(t) \leq M_{1} \exp \left(\int_{\alpha}^{t} b(s) d s\right)+\int_{\alpha}^{t} a^{\prime}(s) \exp \left(\int_{s}^{t} b(\sigma) d \sigma\right) d s,
$$

for $t \in I$, where

$$
M_{1}=\frac{1}{1-p_{1}}\left[a(\alpha)+\int_{\alpha}^{\beta} c(s)\left(\int_{\alpha}^{s} a^{\prime}(\tau) \exp \left(\int_{\tau}^{s} b(\sigma) d \sigma\right) d \tau\right) d s\right]
$$

$\left(\mathrm{a}_{2}\right)$ Suppose that

$$
u(t) \leq a(t)+b(t) \int_{\alpha}^{t} f(s) u(s) d s+c(t) \int_{\alpha}^{\beta} g(s) u(s) d s
$$

for $t \in I$. If

$$
p_{2}=\int_{\alpha}^{\beta} g(s) K_{2}(s) d s<1
$$

then

$$
u(t) \leq K_{1}(t)+M_{2} K_{2}(t)
$$

for $t \in I$, where

$$
\begin{aligned}
& K_{1}(t)=a(t)+b(t) \int_{\alpha}^{t} f(\tau) a(\tau) \exp \left(\int_{\tau}^{t} f(\sigma) b(\sigma) d \sigma\right) d \tau, \\
& K_{2}(t)=c(t)+b(t) \int_{\alpha}^{t} f(\tau) c(\tau) \exp \left(\int_{\tau}^{t} f(\sigma) b(\sigma) d \sigma\right) d \tau
\end{aligned}
$$

and

$$
M_{2}=\frac{1}{1-p_{2}} \int_{\alpha}^{\beta} g(s) K_{1}(s) d s .
$$

$\left(\mathrm{a}_{3}\right)$ Let $h(t, s)$ and its partial derivative $\frac{\partial}{\partial t} h(t, s)$ be nonnegative and continuous functions for $\alpha \leq s \leq t \leq \beta$ and

$$
u(t) \leq a(t)+\int_{\alpha}^{t} h(t, s) u(s) d s+\int_{\alpha}^{\beta} c(s) u(s) d s
$$


for $t \in I$. If

$$
p_{3}=\int_{\alpha}^{\beta} c(s) \exp \left(\int_{\alpha}^{s} B(\sigma) d \sigma\right) d s<1
$$

then

$$
u(t) \leq a(t)+M_{3} \exp \left(\int_{\alpha}^{t} B(\sigma) d \sigma\right)+\int_{\alpha}^{t} A(s) \exp \left(\int_{s}^{t} B(\sigma) d \sigma\right) d s,
$$

for $t \in I$, where

$$
\begin{aligned}
& A(t)=h(t, t) a(t)+\int_{\alpha}^{t} \frac{\partial}{\partial t} h(t, s) a(s) d s, \\
& B(t)=h(t, t)+\int_{\alpha}^{t} \frac{\partial}{\partial t} h(t, s) d s,
\end{aligned}
$$

and

$$
M_{3}=\frac{1}{1-p_{3}} \int_{\alpha}^{\beta} c(s)\left[a(s)+\int_{\alpha}^{s} A(\tau) \exp \left(\int_{\tau}^{s} B(\sigma) d \sigma\right) d \tau\right] d s .
$$

Remark 1. If we take $a(t)=d$ (a constant) and hence $a^{\prime}(t)=0$, then the inequality established in $\left(\mathrm{a}_{1}\right)$ reduces to the slight variant of the inequality given by Bainov and Simeonov in $[2, \mathrm{p} .11]$. The inequality established in $\left(\mathrm{a}_{2}\right)$ is a variant of the inequality given by Gamidov in [4, Lemma 1.2]. By taking $c(t)=0$ in the inequality given in $\left(\mathrm{a}_{3}\right)$, we recapture the inequality given in Theorem 1.8 in $[2$, p.11].

The discrete analogues of the inequalities in Theorem 1 are given in the following theorem.

Theorem 2. Let $u, a, b, c, f, g$ be real-valued nonnegative functions on $N_{\alpha, \beta}$.

$\left(\mathrm{b}_{1}\right)$ Suppose that $\Delta a(n) \geq 0$ for $n \in N_{\alpha, \beta}$ and

$$
u(n) \leq a(n)+\sum_{s=\alpha}^{n-1} b(s) u(s)+\sum_{s=\alpha}^{\beta} c(s) u(s),
$$

for $n \in N_{\alpha, \beta}$. If

$$
q_{1}=\sum_{s=\alpha}^{\beta} c(s) \prod_{\tau=\alpha}^{s-1}[1+b(\tau)]<1,
$$

then

$$
u(n) \leq N_{1} \prod_{s=\alpha}^{n-1}[1+b(s)]+\sum_{s=\alpha}^{n-1} \Delta a(s) \prod_{\sigma=s+1}^{n-1}[1+b(\sigma)],
$$

for $n \in N_{\alpha, \beta}$, where

$$
N_{1}=\frac{1}{1-q_{1}}\left[a(\alpha)+\sum_{s=\alpha}^{\beta} c(s) \sum_{\tau=\alpha}^{s-1} \Delta a(\tau) \prod_{\sigma=\tau+1}^{s-1}[1+b(\sigma)]\right] .
$$


$\left(\mathrm{b}_{2}\right)$ Suppose that

$$
u(n) \leq a(n)+b(n) \sum_{s=\alpha}^{n-1} f(s) u(s)+c(n) \sum_{s=\alpha}^{\beta} g(s) u(s),
$$

for $n \in N_{\alpha, \beta}$. If

$$
q_{2}=\sum_{s=\alpha}^{\beta} g(s) L_{2}(s)<1
$$

then

$$
u(n) \leq L_{1}(n)+N_{2} L_{2}(n)
$$

for $n \in N_{\alpha, \beta}$, where

$$
\begin{aligned}
& L_{1}(n)=a(n)+b(n) \sum_{s=\alpha}^{n-1} f(s) a(s) \prod_{\sigma=s+1}^{n-1}[1+f(\sigma) b(\sigma)], \\
& L_{2}(n)=c(n)+b(n) \sum_{s=\alpha}^{n-1} f(s) c(s) \prod_{\sigma=s+1}^{n-1}[1+f(\sigma) b(\sigma)],
\end{aligned}
$$

and

$$
N_{2}=\frac{1}{1-q_{2}} \sum_{s=\alpha}^{\beta} g(s) L_{1}(s) .
$$

$\left(\mathrm{b}_{3}\right)$ Let $r(n, s), \Delta_{n} r(n, s)=r(n+1, s)-r(n, s)$ be real-valued nonnegative functions for $\alpha \leq s \leq n \leq \beta$ and

$$
u(n) \leq a(n)+\sum_{s=\alpha}^{n-1} r(n, s) u(s)+\sum_{s=\alpha}^{\beta} c(s) u(s),
$$

for $n \in N_{\alpha, \beta}$. If

$$
q_{3}=\sum_{s=\alpha}^{\beta} c(s) \prod_{\tau=\alpha}^{s-1}[1+\bar{B}(\tau)]<1
$$

then

$$
u(n) \leq a(n)+N_{3} \prod_{s=\alpha}^{n-1}[1+\bar{B}(s)]+\sum_{s=\alpha}^{n-1} \bar{A}(s) \prod_{\sigma=s+1}^{n-1}[1+\bar{B}(\sigma)]
$$

for $n \in N_{\alpha, \beta}$, where

$$
\begin{array}{r}
\bar{A}(n)=r(n+1, n) a(n)+\sum_{s=\alpha}^{n-1} \Delta_{n} r(n, s) a(s), \\
\bar{B}(n)=r(n+1, n)+\sum_{s=\alpha}^{n-1} \Delta_{n} r(n, s),
\end{array}
$$


and

$$
N_{3}=\frac{1}{1-q_{3}} \sum_{s=\alpha}^{\beta} c(s)\left[a(s)+\sum_{\tau=\alpha}^{s-1} \bar{A}(\tau) \prod_{\sigma=\tau+1}^{s-1}[1+\bar{B}(\sigma)]\right]
$$

Remark 2. By taking $c(n)=0$ in $\left(\mathrm{b}_{1}\right)$ we get the inequality given in Theorem 1.2.6 in [7]. The inequalities in $\left(b_{2}\right)$ and $\left(b_{3}\right)$ can be considered as the useful variants of the inequalities in Theorem 1.2.3 and Theorem 1.3.4 given in [7].

\section{Proofs of Theorems 1 and 2}

$\left(\mathrm{a}_{1}\right)$ Define a function $z(t)$ by the right hand side of $(2.1)$. Then $u(t) \leq z(t)$,

$$
z(\alpha)=a(\alpha)+\int_{\alpha}^{\beta} c(s) u(s) d s
$$

and

$$
z^{\prime}(t)=a^{\prime}(t)+b(t) u(t) \leq a^{\prime}(t)+b(t) z(t)
$$

which implies

$$
u(t) \leq z(t) \leq z(\alpha) \exp \left(\int_{\alpha}^{t} b(\sigma) d \sigma\right)+\int_{\alpha}^{t} a^{\prime}(s) \exp \left(\int_{s}^{t} b(\sigma) d \sigma\right) d s .
$$

From (3.1) and (3.2) we have

$$
z(\alpha) \leq a(\alpha)+\int_{\alpha}^{\beta} c(s)\left\{z(\alpha) \exp \left(\int_{\alpha}^{s} b(\sigma) d \sigma\right)+\int_{\alpha}^{s} a^{\prime}(\tau) \exp \left(\int_{\tau}^{s} b(\sigma) d \sigma\right) d \tau\right\} d s
$$

i.e.

$$
\begin{aligned}
& z(\alpha)\left\{1-\int_{\alpha}^{\beta} c(s) \exp \left(\int_{\alpha}^{s} b(\sigma) d \sigma\right) d s\right\} \\
\leq & a(\alpha)+\int_{\alpha}^{\beta} c(s)\left(\int_{\alpha}^{s} a^{\prime}(\tau) \exp \left(\int_{\tau}^{s} b(\sigma) d \sigma\right) d \tau\right) d s,
\end{aligned}
$$

which implies

$$
z(\alpha) \leq M_{1}
$$

Using (3.3) and (3.2) we get the desired inequality in (2.3).

$\left(\mathrm{a}_{2}\right)$ Let

$$
\begin{aligned}
z(t) & =\int_{\alpha}^{t} f(s) u(s) d s, \\
k & =\int_{\alpha}^{\beta} g(s) u(s) d s .
\end{aligned}
$$


Then $z(\alpha)=0,(2.5)$ can be restated as

$$
u(t) \leq a(t)+b(t) z(t)+c(t) k,
$$

and

$$
z^{\prime}(t)=f(t) u(t)
$$

From (3.7) and (3.6) we have

$$
z^{\prime}(t) \leq\{f(t) a(t)+k f(t) c(t)\}+f(t) b(t) z(t),
$$

which implies

$$
z(t) \leq \int_{\alpha}^{t}\{f(\tau) a(\tau)+k f(\tau) c(\tau)\} \exp \left(\int_{\tau}^{t} f(\sigma) b(\sigma) d \sigma\right) d \tau
$$

Using (3.8) in (3.6) we get

$$
\begin{aligned}
u(t) & \leq\{a(t)+k c(t)\}+b(t) \int_{\alpha}^{t}\{f(\tau) a(\tau)+k f(\tau) c(\tau)\} \exp \left(\int_{\tau}^{t} f(\sigma) b(\sigma) d \sigma\right) d \tau \\
& =K_{1}(t)+k K_{2}(t) .
\end{aligned}
$$

From (3.5), (3.9) and in view of (2.6), as in the proof of $\left(\mathrm{a}_{1}\right)$, it is easy to observe that

$$
k \leq M_{2}
$$

Using (3.10) in (3.9) we get (2.7).

$\left(\mathrm{a}_{3}\right)$ Define a function $z(t)$ by

$$
z(t)=\int_{\alpha}^{t} h(t, s) u(s) d s+\int_{\alpha}^{\beta} c(s) u(s) d s .
$$

Then, (2.11) can be restated as

$$
\begin{aligned}
& u(t) \leq a(t)+z(t), \\
& z(\alpha)=\int_{\alpha}^{\beta} c(s) u(s) d s .
\end{aligned}
$$

and

$$
\begin{aligned}
z^{\prime}(t) & =h(t, t) u(t)+\int_{\alpha}^{t} \frac{\partial}{\partial t} h(t, s) u(s) d s \\
& \leq h(t, t)\{a(t)+z(t)\}+\int_{\alpha}^{t} \frac{\partial}{\partial t} h(t, s)\{a(s)+z(s)\} d s \\
& \leq A(t)+B(t) z(t),
\end{aligned}
$$


which implies

$$
z(t) \leq z(\alpha) \exp \left(\int_{\alpha}^{t} B(\sigma) d \sigma\right)+\int_{\alpha}^{t} A(s) \exp \left(\int_{s}^{t} B(\sigma) d \sigma\right) d s .
$$

The rest of the proof can be completed by following the proofs of $\left(\mathrm{a}_{1}\right),\left(\mathrm{a}_{2}\right)$ given above.

$\left(\mathrm{b}_{1}\right)$ Define a function $z(n)$ by the right hand side of $(2.17)$. Then $u(n) \leq z(n)$,

$$
z(\alpha)=a(\alpha)+\sum_{s=\alpha}^{\beta} c(s) u(s)
$$

and

$$
\Delta z(n)=\Delta a(n)+b(n) u(n) \leq \Delta a(n)+b(n) z(n) .
$$

Now a suitable application of Theorem 1.2.1 given in [7] to (3.16) yields

$$
u(n) \leq z(n) \leq z(\alpha) \prod_{s=\alpha}^{n-1}[1+b(s)]+\sum_{s=\alpha}^{n-1} \Delta a(s) \prod_{\sigma=s+1}^{n-1}[1+b(\sigma)] .
$$

From (3.15), (3.17) and in view of (2.18) we have

$$
z(\alpha) \leq N_{1}
$$

Using (3.18) in (3.17) we get the required inequality in (2.19).

The proofs of $\left(b_{2}\right)$ and $\left(b_{3}\right)$ follows by closely looking at the proofs of $\left(a_{2}\right),\left(a_{3}\right)$ and $\left(b_{1}\right)$ (see also [7]). Here we leave the details to the reader.

\section{Some Applications}

In this section, we present applications of the inequality in Theorem 1, part $\left(\mathrm{a}_{2}\right)$ to study certain properties of the solutions of Volterra-Fredholm integral equation

$$
x(t)=e(t)+\int_{\alpha}^{t} F(t, s, x(s)) d s+\int_{\alpha}^{\beta} G(t, s, x(s)) d s,
$$

for $t \in I$, where $x(t)$ is an unknown function, $e \in C(I, R), F, G \in C(E \times R, R)$. Here we note that the existence proofs for the solution of equation (4.1) show either that the operator $T$ defined by the right hand side of (4.1) is a contraction (in which case one also has uniqueness) or $T$ is compact and continuous on a suitable subspace of the space of continuous functions (see also $[1,5]$ ).

The following theorem deals with the estimate on the solution of equation (4.1).

Theorem 3. Suppose that the functions e, F, G in equation (4.1) satisfy the conditions

$$
\begin{aligned}
|e(t)| & \leq a(t), \\
|F(t, s, x)| & \leq b(t) f(s)|x|, \\
|G(t, s, x)| & \leq c(t) g(s)|x|
\end{aligned}
$$


where $a, b, c, f, g$ are as defined in Theorem 1. Let $p_{2}$ be as in (2.6). If $x(t)$ is a solution of equation (4.1) on $I$, then

$$
|x(t)| \leq K_{1}(t)+M_{2} K_{2}(t)
$$

for $t \in I$, where $K_{1}, K_{2}, M_{2}$ are as given in Theorem 1 , part $\left(\mathrm{a}_{2}\right)$.

Proof. Let $x(t)$ be a solution of equation (4.1) on $I$. Using the fact that $x(t)$ is a solution of equation (4.1) and (4.2)-(4.4) we have

$$
|x(t)| \leq a(t)+b(t) \int_{\alpha}^{t} f(s)|x(s)| d s+c(t) \int_{\alpha}^{\beta} g(s)|x(s)| d s .
$$

Now an application of the inequality in Theorem 1, part $\left(\mathrm{a}_{2}\right)$ to (4.6) yields the required estimate in (4.5).

The next result deals with the uniqueness of solutions of equation (4.1).

Theorem 4. Suppose that the functions $F, G$ in equation (4.1) satisfy the conditions

$$
\begin{gathered}
|F(t, s, x)-F(t, s, \bar{x})| \leq b(t) f(s)|x-\bar{x}|, \\
|G(t, s, x)-G(t, s, \bar{x})| \leq c(t) g(s)|x-\bar{x}|,
\end{gathered}
$$

where $b, c, f, g$ are as in Theorem 1. Let $p_{2}$ be as in (2.6). Then the equation (4.1) has at most one solution on $I$.

Proof. Let $u(t)$ and $v(t)$ be two solutions of (4.1) on $I$. Using the facts that $u(t)$ and $v(t)$ are the solutions of (4.1) on $I$ and (4.7), (4.8) we have

$$
|u(t)-v(t)| \leq b(t) \int_{\alpha}^{t} f(s)|u(s)-v(s)| d s+c(t) \int_{\alpha}^{\beta} g(s)|u(s)-v(s)| d s .
$$

Now an application of the inequality given in Theorem 1, part $\left(\mathrm{a}_{2}\right)$ (with $a(t)=0$ which in fact implies $\left.K_{1}(t)=0, M_{2}=0\right)$ to (4.9) yields $u(t)=v(t)$, i.e. there is at most one solution of equation (4.1) on $I$.

Remark 3. We note that the inequality given in Theorem 2 , part $\left(b_{2}\right)$ can be used to study similar properties as in Theorems 3 and 4 for the solutions of sum-difference equation

$$
z(n)=e(n)+\sum_{s=\alpha}^{n-1} F(n, s, z(s))+\sum_{s=\alpha}^{\beta} G(n, s, z(s)),
$$

for $n \in N_{\alpha, \beta}$, under some suitable conditions on the functions involved in (4.10). For various other applications of the inequalities of the type given here, see [2, 3, 6-10] and some of the references cited therein. 


\section{References}

[1] S. Asirov and Ja. D. Mamedov, Investigation of solutions of nonlinear Volterra-Fredholm operator equations, Dokl. Akad. Nauk SSSR 229(1976), 982-986.

[2] D. Bainov and P. Simeonov, Integral Inequalities and Applications, Kluwer Academic Publishers, Dordrecht, 1992.

[3] S. S. Dragomir, Some Gronwall type inequalities and Applications, Nova Science Publishers, Inc. New York, 2003.

[4] Sh. G. Gamidov, Integral inequalities for boundary value problems for differential equations, Diff. Eq. 5(1969), 463-472 (English Translation from Russian).

[5] R. K. Miller, J. A. Nohel and J. S. W. Wong, A stability theorem for nonlinear mixed integral equations, J. Math. Anal. Appl. 25(1969), 446-449.

[6] B. G. Pachpatte, Inequalities for Differential and Integral Equations, Academic Press, New York, 1998.

[7] B. G. Pachpatte, Inequalities for Finite Difference Equtions, Marcel Dekker,Inc., New York, 2002.

[8] B. G. Pachpatte, A note on certain integral inequality, Tamkang J. Math. 33(2002), 353358.

[9] B. G. Pachpatte, On an inequality applicable to general Volterra-Fredholm integral equation, An. Sti. Univ. Al. I. Cuza Iasi, Mat. XLIX(2003), 221-228.

[10] B. G. Pachpatte, Explicit bound on a retarded integral inequality, Math. Ineq. Appl. 7(2004), 7-11.

57 Shri Niketan Colony, Near Abhinay Talkies, Aurangabad 431001 (Maharashtra) India.

E-mail: bgpachpatte@hotmail.com 